\title{
Long-term stable hydrogels for biorelevant dissolution testing of drug-eluting stents
}

\author{
Beatrice Semmling ${ }^{1}$, Stefan Nagel ${ }^{1}$, Katrin Sternberg ${ }^{2}$, Werner Weitschies ${ }^{1}$ and Anne Seidlitz ${ }^{1 *}$ \\ ${ }^{*}$ Correspondence: anne.seidlitz@uni-greifswald.de \\ 'Institute of Pharmacy, Center of Drug Absorption and Transport, University of Greifswald, Germany. \\ 2Institute for Biomedical Engineering, University of Rostock, Germany.
}

\begin{abstract}
Background: For the purpose of biorelevant dissolution testing hydrogels have recently been used to investigate release and distribution behavior of drugs released from specialized dosage forms such as drug-eluting stents. For stent testing using the vesselsimulating flow-through cell (vFTC) certain functional properties regarding life time of the used hydrogels such as hardening/ softening, degradation/erosion and swelling/shrinkage during the time period of dissolution testing are of highest interest.

Methods: Hydrogels composed of alginate, agar, agarose, polyacrylamide (PAA) and poly(vinyl alcohol) (PVA) were prepared using physical and chemical cross-linking methods. To characterize the mechanical stability of the test specimens stress-strain curves were recorded by texture analysis before and after perfusion in the vFTC for 28 days and Young's moduli were calculated. The surface morphology of the test specimens was examined using scanning electron microscopy. Water uptake upon incubation was determined.

Results: Besides the previously established alginate gels, suitable hydrogels consisting of $2 \mathrm{wt} \%$ agar, 2 wt $\%$ agarose, $10 \mathrm{wt} \%$ PAA or $15 \mathrm{wt} \%$ PVA were identified. Comparison of stress-strain curves indicated a sample softening of reference as well as agar and a slight hardening of PVA whereas hardness of agarose and PAA remained unchanged. Young's moduli of agarose and PAA were almost unaffected after 28 days of perfusion. Swelling of PAA by $18 \mathrm{wt} \%$ and shrinkage of PVA by $14 \mathrm{wt} \%$ was observed compared to agar and agarose whose water uptake was negligibly small.

Conclusion: The hydrogels differ not only in their preparation procedure but also regarding their swelling properties, surface morphology and texture. Due to the ease of handling, mild gelling conditions, no swelling tendencies, elastic properties, and nearly unaffected texture after 28 days of perfusion compared to native test specimens, agarose gels are considered as the best choice for longterm testing using the vFTC.
\end{abstract}

Keywords: Hydrogel, alginate, agar, agarose, polyacrylamide, drug-eluting stent, in vitro dissolution testing, vessel-simulating flowthrough cell

\section{Introduction}

Hydrogels consist of natural (e.g. alginate, collagen, agarose, agar) or synthetic (e.g. polyacrylamide/poly(acrylic acid) blends, poly(methacrylate), poly(ethylene oxide), poly(vinyl alcohol)) polymers forming insoluble three-dimensional scaffolds which enable the embedding of large amounts of aqueous media or body fluids. The ability to absorb and retain fluids leads to material properties such as high water content and elasticity which are comparable to some physico-chemical properties of soft tissue. Swelling behavior is directly related to the chemical structure of the polymer and its cross-linking density. Gel-formation is induced by various stimuli-responsive strategies such as target antigens [1], specific ions such as $\mathrm{Ca}^{2+}$ $[2,3]$, temperature and $\mathrm{pH}$ changes [4], UV irradiation or the addition of cross-linking agents such as radical starters [3,5].

Hydrogels are of increasing importance for biomedical applications $[\mathbf{6}, 7]$. They are used for cell encapsulation [8], tissue engineering [9] and in controlled release dosage forms $[10,11]$. Recently, hydrogels have been used for biorelevant dissolution testing of dosage forms which are injected or implanted into tissue [12-16] to simulate some properties of the tissue surrounding the drug delivery systems such as drug transport via diffusion. Whereas convective drug transport dominates in fully-agitated dissolution test setups diffusion is the predominant mechanism of drug transport in hydrogels. This change in the predominant transport characteristic has been shown to impact drug release in different ways. While Hoang Thi et al., [13] and Seidlitz et al., [14] observed a slower release in the diffusion-controlled setup using a gel compartment, Klose et al., [12] report on a faster release using their gel-embedding method. However, in this case the drug was released faster due to local changes in $\mathrm{pH}$ caused by released polymer fragments. This phenomenon did not occur in the fully-agitated reference setup. These studies illustrate how diverse certain parameters of the test design may influence the results of the release study. In this context, it is noteworthy, that up to date no specific dissolution test methods (whether biorelevant or not) have been monographed for the examination of release from parenteral dosage forms in the United States Pharmacopeia as well as in the European or Japanese Pharmacopoeia.

The vessel-simulating flow-through cell (vFTC) $[14,16]$ has been developed in order to examine drug release and

(c) 2013 Seidlitz et al; licensee Herbert Publications Ltd. This is an Open Access article distributed under the terms of Creative Commons Attribution License (http://creativecommons.org/licenses/by/3.0). This permits unrestricted use, distribution, and reproduction in any medium, provided the original work is properly cited. 
Semmling et al. Journal of Pharmaceutical Technology \& Drug Research 2013,

distribution from drug-eluting stents (DES) under conditions adapted to some of the aspects of the in vivo stent placement. The VFTC is based on the compendial flow-through cell which is described in the respective Pharmacopeias. The chamber of the vFTC was previously equipped with an alginate hydrogel with a central opening into which a DES may be implanted. This setup allows for the examination of the resulting transport forces on the luminal side by convection (flowing media) and on the abluminal side by diffusion (simulated vessel wall).

However, changes in functional gel properties after long exposure to the test conditions limit the period of use of gels as an acceptor compartment in release and distribution studies. For example, polymer degradation (and consequently erosion of the gel) may lead to a decrease of the gel volume. Hydrogel swelling or shrinkage may result in varying water content and volume of the gel. Hardness and elasticity of the gel may vary with increasing perfusion time. Such changes in the gel properties may also influence the dissolution tests. These phenomena may be problematic when performing dissolution tests with DES which are expected to release their drug load over a time span of up to 90 days. Even though the impact of a gel compartment on the outcome of the dissolution test is expected to be negligible when the release rate is very low (e.g. release from the implant is much slower than diffusion through the gel), it is desirable for dissolution testing of such a dosage form to conduct the tests until at least $80 \%$ of the drug load have been released as recommended by the Food and Drug Administration (FDA) for solid oral dosage forms (FDA) [17]. This has been reported to be the case in a porcine coronary artery model for example for the FDA approved CYPHER stent and for the NEVO stent (Cordis Corporation, Johnson \& Johnson, Warren, NJ) [18] and also in humans for the CYPHER stent within 30 days $[19,20]$, respectively. Therefore a suitable hydrogel should be functional for at least 30 days. The currently used alginate gel is not suitable for such long-term studies since the replacement of the gelling $\mathrm{Ca}^{2+}$ ions by monovalent buffer ions is inevitable and leads to gel liquefaction. Further target parameters for an "ideal" gel compartment for the vFTC are: short time spans for sol-gel transition or, generally, short time spans for gel preparation, transparency to facilitate DES placement, form-stability to retain the cylindrical shape of gelled samples (especially to ensure the constant opening of the hydrogel lumen) based on the gel resistance to media perfusion (shear stress), elasticity to allow for stent implantation and balloon catheter inflation, no distinct polymer degradation/gel erosion, negligible swelling / shrinkage to exclude changes in the dimension of the vessel-simulating lumen, diffusibility allowing for drug distribution into the gel, and biocompatibility to optionally allow for the integration of living cells.

Numerous studies have been performed on the characterization of the mechanical properties of gelled foods, solid dosage forms or hydrogels used for medical or pharmaceutical applications $[3, \mathbf{8}, \mathbf{2 1}, \mathbf{2 2}]$. However, data regarding long-term stability of gels is sparse. The purpose of this study was to identify suitable long-term stable hydrogel formulations for dissolution testing of DES using the vFTC and to characterize them regarding their long-term stability. The prepared test gels were assessed based on the ease of preparation technique and evaluated both optically and by scanning electron microscopy (SEM) after the gel samples were freeze-dried to capture characteristics of the sample surface morphology. In order to find a measure for the long-term stability of the gels, mechanical properties of the prepared gels were determined by texture analysis. For this purpose stress-strain curves of native gels as well as gels that had been perfused with phosphate buffered saline (PBS) pH 7.4 in the VFTC for 28 days were recorded. To further study gel elasticity Young's moduli were calculated and the water uptake of prepared gels was examined.

\section{Materials and methods}

Agar (gel point $34-37^{\circ} \mathrm{C}$ ) and agarose (gel point $36^{\circ} \mathrm{C} \pm 1.5^{\circ} \mathrm{C}$ ) were purchased from Sigma-Aldrich (Steinheim, Germany). Ammonium peroxodisulphate (APS, $\geq 98 \%$, p.a., $A C S$ ), $N, N_{,}, N^{\prime}, N^{\prime}-$ tetramethylethylenediamine (TEMED, 99\%, p.a., for gel electrophoresis) and Rotiphorese gel 30 (aqueous $30 \mathrm{wt} \%$ acrylamide/ $\mathrm{N}, \mathrm{N}^{\prime}$-methylene-bis-acrylamide stock solution with a ratio of 37.5:1) were purchased from Carl Roth GmbH \& Co. KG (Karlsruhe, Germany). Fully hydrolyzed PVA (degree of hydrolysis $\geq 98 \%$ ) of a molecular weight of $145.000 \mathrm{~g} / \mathrm{mol}$ and $60.000 \mathrm{~g} /$ mol, respectively, were purchased from Merck Schuchardt OHG (Hohenbrunn, Germany). PVA of a molecular weight of $72.000 \mathrm{~g} / \mathrm{mol}$ was purchased from AppliChem GmbH (Darmstadt, Germany). Sodium alginate was purchased from Fagron GmbH \& Co. KG (Barsbüttel, Germany).

\section{Preparation of perfusion media}

PBS pH 7.4 was prepared in accordance with the European Pharmacopoeia by dissolving $2.38 \mathrm{~g}$ disodium hydrogen phosphate dodecahydrate, $0.19 \mathrm{~g}$ potassium dihydrogen phosphate and $8.00 \mathrm{~g}$ sodium chloride in $1000 \mathrm{~mL}$ purified water. If necessary, buffer $\mathrm{pH}$ was adjusted to $\mathrm{pH} 7.4 \pm 0.5$ using the respective buffer salt.

\section{Preparation of test specimen Reference gel}

Alginate gels were prepared as previously reported [14]. Briefly, $3 \mathrm{~g}$ sodium alginate powder were dissolved in $100 \mathrm{~mL}$ purified water. $16.5 \mathrm{~g}$ sodium alginate solution were gelled with $1.65 \mathrm{~g}$ of a suspension of calcium sulfate in purified water ( $9 \mathrm{wt} \%)$ and $470 \mathrm{~mL}$ of $10 \mathrm{wt} \%$ sodium phosphate $\left(\mathrm{Na}_{3} \mathrm{PO}_{4} \times 12 \mathrm{H}_{2} \mathrm{O}\right)$ solution by using an internal gelation method [2].

\section{Agar gel}

To prepare $2 \mathrm{wt} \%$ agar gels, $2 \mathrm{~g}$ agar powder were dissolved in $100 \mathrm{~g} 90^{\circ} \mathrm{C}$ hot, purified water under stirring. Evaporated water was replenished during cooling at a temperature of 
approximately $50^{\circ} \mathrm{C}$. Prior to gelation the polymer solution was cast into a vFTC and was allowed to solidify at room temperature.

\section{Agarose gel}

To prepare $2 \mathrm{wt} \%$ agarose gels, $2 \mathrm{~g}$ agarose powder were dissolved in $100 \mathrm{~g} 90^{\circ} \mathrm{C}$ hot, purified water under stirring. Evaporated water was replenished during cooling at a temperature of approximately $50^{\circ} \mathrm{C}$. Prior to gelation the polymer solution was cast into a vFTC and was allowed to solidify at room temperature.

\section{Polyacrylamide gel}

PAA gels were composed of a blend of $6.7 \mathrm{~g}$ of Rotiphorese gel 30 and $12.9 \mathrm{~g}$ of PBS pH 7.4. Radical polymerization of the acrylamide was initiated by the addition of $200 \mu \mathrm{L}$ of an aqueous $10 \mathrm{wt} \%$ APS solution. $20 \mu \mathrm{L}$ TEMED were added acting as an additional catalyst for the polymerization process. Subsequently the mixture was homogenized, poured into the vFTC and allowed to gel.

\section{Poly(vinyl alcohol) gel}

Solutions of PVA (with varying molecular weight: $60.000 \mathrm{~g} /$ $\mathrm{mol}, 72.000 \mathrm{~g} / \mathrm{mol}$ and $145.000 \mathrm{~g} / \mathrm{mol}$ ) were prepared by dissolving the polymer powder in boiling, purified water. For each molecular weight PVA solution with concentrations of 6 wt $\%, 10 \mathrm{wt} \%, 15 \mathrm{wt} \%$ and $20 \mathrm{wt} \%$ were prepared. Physically cross-linked gels were prepared using a freeze-thaw technique (cryogelation) $[\mathbf{2 3}, \mathbf{2 4}]$. Samples of prepared PVA solutions were poured into custom-designed cylindrical moulds formed of polysiloxane (EN ISO 4823, type 0) with identical measures as the vFTC. Subsequently, samples were solidified inside the moulds by freezing the PVA solution at $-80^{\circ} \mathrm{C}$ over night and by thawing at $4{ }^{\circ} \mathrm{C}$ for $5 \mathrm{~h}$ conducting $4,5,7$ or 10 freeze-thaw cycles. After solidification the samples were removed from the mould and placed in the vFTC.

\section{Morphological characterization}

Prepared gel cylinders were evaluated optically, thus transparency, air inclusions as well as cracks in the reference and test gels after gel-formation were studied. The time required for solidification and preparation practicability was recorded.

For SEM gels were prepared as described above. After cooling petroleum with liquid nitrogen to $-80^{\circ} \mathrm{C}$ the gels were immersed within this liquid for $60 \mathrm{~s}$. Frozen samples were freeze-dried with a Christ alpha 2-4 freeze-dryer (Martin Christ Gefriertrocknungsanlagen $\mathrm{GmbH}$, Osterode am Harz, Germany) at $5{ }^{\circ} \mathrm{C}$ and 0.1 mbar. For SEM imaging the freeze-dried samples were fractured, fixed on specimen stubs and stored in a desiccator which was evacuated to a pressure $\leq 10 \mathrm{mbar}$. Lyophilisates were sputtered with a thin layer of gold-palladium (mini sputter coater SC7620, Quorum Technologies Ltd., West Sussex, UK) in an argon atmosphere for $90 \mathrm{~s}$ and imaged by SEM (Phenom, FEI Company, L.O.T.-
Oriel GmbH \& Co. KG, Darmstadt, Germany).

\section{Degree of swelling}

For the determination of equilibrium swelling the water uptake of the reference and test gels was determined by incubating test specimen in $100 \mathrm{~mL}$ PBS pH 7.4 at $37^{\circ} \mathrm{C}$ for three days. The hydrogels were prepared as described above. After solidification in the vFTC the gelled samples were removed, weighed and transferred into flasks containing buffered media. At the end of the experiment the gels were removed, carefully dabbed thus removing adherent liquid with soft, lint-free paper and reweighed. The water uptake (\%) was calculated using equation 1 :

$$
\text { Degree of swelling }(\%)=\frac{w_{b}-w_{a}}{w_{a}} * 100 \quad \text { Equation (1) }
$$

in which $w_{a}$ is the initial weight of the gel sample and $w_{b}$ the weight of the swollen gel sample after incubation, respectively.

\section{Investigation of long-term stability}

Mechanical stability of the reference and test gels was examined by texture analysis after continuous perfusion within the vFTC over a period of 28 days. A schematic overview of the $\mathrm{vFTC}$ is given in (Figure 1).

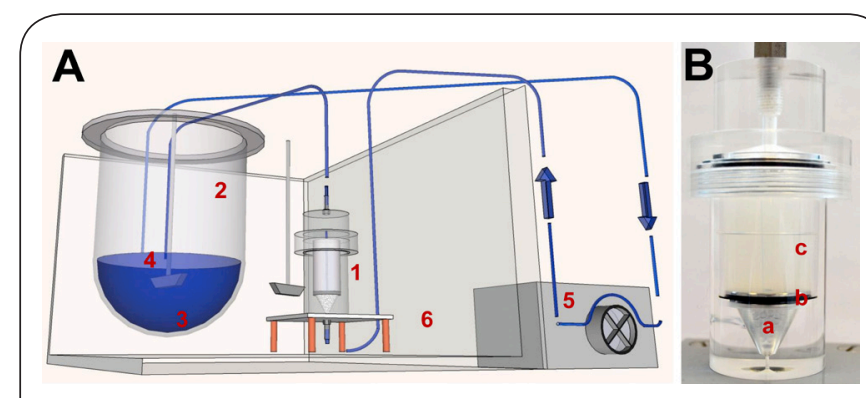

Figure 1. Schematic overview of the in vitro test setup (A) and photograph of the vFTC equipped with a $2 \mathrm{wt} \%$ agarose gel (B); 1) vFTC, 2) media container, 3) PBS of $\mathrm{pH} 7.4$, 4) paddle stirred at $50 \mathrm{rpm}, 5)$ peristaltic pump, 6) heated water bath, a) glass beads, b) stainless steel disc, c) hydrogel.

The VFTC was equipped either with the reference or one of the test gels. For a detailed description of the vFTC see [14]. The placeholder rod which was placed in the gel during solidification was removed, top and bottom part of the vFTC were tightly screwed and the vFTC was positioned within a heated water bath $\left(37 \pm 0.5^{\circ} \mathrm{C}\right)$. Phosphate-buffered saline with a $\mathrm{pH}$ of 7.4 was used as the perfusion media. The flow-rate was set to $35 \mathrm{~mL} / \mathrm{min}$ [25] and the media was continuously pumped through the gel lumen from the bottom to the top in a closed loop configuration. During longer incubation experiments the perfusion media was periodically replaced by freshly prepared PBS pH 7.4 to avoid microbial contamination. 
Semmling et al. Journal of Pharmaceutical Technology \& Drug Research 2013,

http://www.hoajonline.com/journals/pdf/2050-120X-2-19.pdf

Experiments were terminated at predetermined time points. After removal of the hydrogels from the vFTC the texture properties were measured with the TA.XTPlus texture analyzer (Texture Technologies, Scarsdale, United States of America) on day 1 and 2 for alginate and on day 28 for agar, agarose, PAA and PVA. For comparison, native samples of all gels were prepared (day 0) and examined accordingly. Sample height for TA.XTPlus measurements was $20 \mathrm{~mm}$. Cross-sectional area of the cylindrical gel samples was calculated as the difference between the inner diameter of the vFTC and the diameter of the vessel-simulating lumen and amounted to $4^{*} 10^{-4} \mathrm{~m}^{2}$. A circular plate of a diameter of $40 \mathrm{~mm}$ was used to apply predefined pressure onto the test specimen. Starting distance between top and bottom plate was set to $40 \mathrm{~mm}$. The speed of the plunger was set to a constant value of $2 \mathrm{~mm} / \mathrm{s}$. For the investigation of mechanical properties test specimens of gelled food products are usually compressed by $75-80 \%$ of their original height. These deformation values correspond to reported fracture points since the test specimens will typically break at values of $70-80 \%$ sample compression [26]. Breakage of the gel samples is, however, not mandatory to study mechanical properties. Deformation values below fracture can be applied as recommended for gelled food systems (20-50\%) by Pons and Fiszman [26]. Accordingly, test specimens were compressed by $25 \%$ (reference) and $35 \%$ (test gels) of their original height in this study. Data for force and length deformation were recorded automatically by the data acquisition software (Exponent). In addition, the gels were studied under repeated compression. The time span between the measurements was set to $1 \mathrm{~min}$. Force-time data were reported as stress-strain profiles. Data for compressive strain is presented as the length deformation (\%) of the original height of the gels of $20 \mathrm{~mm}$. Compressive stress data $(\mathrm{kPa})$ were calculated from the initial cross-sectional area of the gel cylinders and the measured force data. Texture analyses were performed in duplicate for each type of gel and one stress-strain curve representative for both test specimens of each gel formulation is reported. The parameter "hardness" was defined as the force which was applied to compress test specimen to the predetermined maximum deformation value, as suggested by Szczesniak et al., [27].

Young's moduli for the reference and test gels were calculated from the linear part of the slope of the first loading loop (0-10\% compression) of the stress-strain curve using equation 2 :

$$
\text { Young 's modulus }(E)=\frac{F / A}{\Delta l / h} \quad \text { Equation (2) }
$$

in which $F(N)$ represents the force applied to the gel cylinder, $A\left(\mathrm{~m}^{2}\right)$ displays the calculated cross-sectional area of the gel cylinder, $\Delta l(m)$ represents the length deformation and $h(m)$ represents the original sample height. Young's modulus $\mathrm{E}$ ( $\mathrm{MPa}$ ) was calculated for each sample and is reported separately for reasons of comparability of the two individually measured stress-strain curves.

\section{Results \\ Preparation techniques and morphological character- ization of test specimen}

The gels prepared varied regarding the choice of polymers, polymer concentrations and mechanism of gel-formation. Photographs of native hydrogels of the final gel formulations obtained immediately after preparation are shown in (Figure 2).

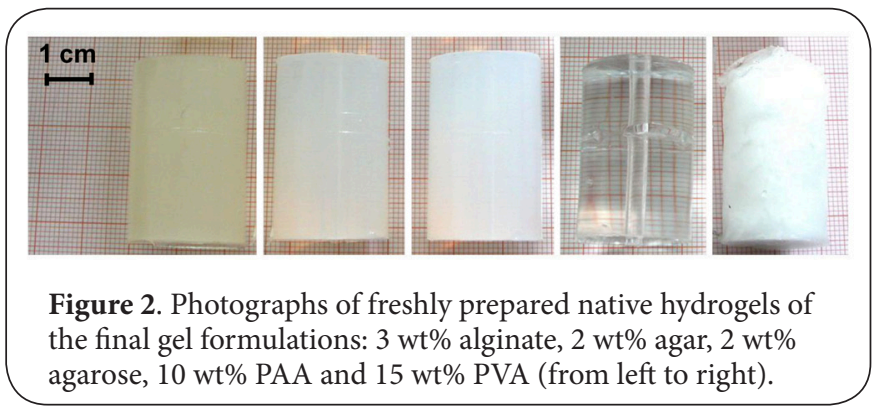

lonically cross-linked alginate samples which served as the reference formulation gelled within $15 \mathrm{~min}$. The reference gels were colored yellow to brownish and were only moderately transparent. The gel formulations containing $2 \mathrm{wt} \%$ agar or agarose resulted in form-stable and slightly turbid but mainly uncolored gels. A concentration of $10 \mathrm{wt} \%$ of Rotiphorese gel 30 was suitable to obtain form-stable PAA samples. Gels containing more than $10 \mathrm{wt} \%$ of Rotiphorese gel 30 were increasingly brittle and thus cracks and fractures within the gels were observed after removal of the placeholder rod. PAA cylinders prepared from $10 \mathrm{wt} \%$ of Rotiphorese gel 30 appeared clear and entirely transparent. The recorded time for gel-formation for PAA was approximately 10 min whereas the time required for solidification of polymer solutions of agar and agarose was between 10 and $15 \mathrm{~min}$. PVA gel-formation was achieved by cryogelation. Form-stability of PVA increased with increasing molecular weight $(60.000 \mathrm{~g} / \mathrm{mol}<72.000 \mathrm{~g} /$ $\mathrm{mol}<145.000 \mathrm{~g} / \mathrm{mol}$ ), with increasing polymer concentration $(6 \mathrm{wt} \%<10 \mathrm{wt} \%<15 \mathrm{wt} \%<20 \mathrm{wt} \%)$ and with an increasing number of freeze-thaw cycles $(1<3<5<7)$. Preparation of the PVA gels took up to 7 days depending on the number of freeze-thaw steps. Only polymer solutions of $145.000 \mathrm{~g} /$ mol with a percentage of $15 \mathrm{wt} \%$ or $20 \mathrm{wt} \%$ which were exposed to 5 or 7 freezing-thawing cycles produced formstable gels. However, the high viscosity of the $20 \mathrm{wt} \%$ solution $(145.000 \mathrm{~g} / \mathrm{mol})$ complicates dosing of the polymer solution. As the numbers of freeze-thaw steps increased, samples were observed to become increasingly whiter. Gel cylinders of sufficient form-stability were found to be non-transparent and completely white marbled with light and dark portions on the surface. Gels obtained from $15 \mathrm{wt} \%$ PVA (145.000 g/ mol) by 5 cryogelation steps were considered most suitable and used for further studies (in the following abbreviated as PVA gels or cryogels). Cryogels showed no signs of gas 
entrapment. The gas entrapment in form of visible air bubbles observed for the other gels was only moderate.

The surface morphology of the reference and test gels was investigated by SEM. A representative selection of SEM images of each gel is given in (Figure 3 ).

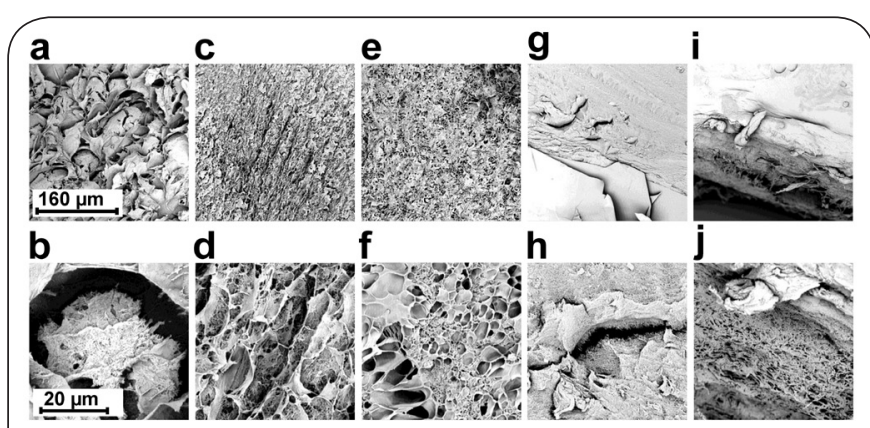

Figure 3. SEM images of hydrogel surfaces of freeze-dried gels at different magnifications (top: 600, bottom: 4000). Hydrogel scaffolds obtained from test gels: $3 \mathrm{wt} \%$ alginate (a, b), 2 wt $\%$ agar (c, d), $2 \mathrm{wt} \%$ agarose (e, f), $10 \mathrm{wt} \%$ PAA (g, h) and $15 \mathrm{wt} \%$ PVA $(\mathbf{i}, \mathbf{j})$.

(Figure 3) a-j illustrates a porous and sponge-like structure formed by the polymeric network. Pores were visible for all gels except for PAA. Examination of higher magnification images of the gels indicated a fine and delicate macrostructure. Larger pores were observed for alginate (b), agar (d) and agarose (f) samples, respectively, whereas on PVA (j) sample surfaces only a few details of its porous network could be identified. For the overall PAA structure no details (pores) were observed.

\section{Degree of swelling}

Liquid absorption has a large impact on sample properties leading to changes in the gel cylinder dimensions or the mechanical behavior. To gain an impression of the swelling properties of the prepared gels the water uptake upon incubation was determined by differential weighing. The results of equilibrium water uptake obtained (Table 1) showed differences from $18.4 \mathrm{wt} \%$ mass gain (PAA) to $14.3 \mathrm{wt} \%$ mass loss (PVA). The tendency of agar and agarose to absorb water was found to be negligible small ( $<1 \mathrm{wt} \%)$. The water uptake for the reference alginate was found to be $21.6 \mathrm{wt} \%$. It is noteworthy that no cracks in the test gels or signs of hydrogel erosion were observed at the end of the experiment. Narrowing of the cylindrical lumen was not observed.

Table 1. Water uptake in percent of the original mass for the reference and the test gels after incubation with PBS of $\mathrm{pH} 7.4$ for 3 days, calculated using equation 1 , data presented as mean \pm SD $(\mathbf{n}=3)$.

\begin{tabular}{lllll}
\hline alginate & agar & agarose & PAA & PVA \\
\hline $21.6 \pm 2.3$ & $0.4 \pm 0.1$ & $0.3 \pm 0.2$ & $18.4 \pm 1.4$ & $-14.3 \pm 2.4$ \\
\hline
\end{tabular}

\section{Long-term stability of test specimen}

To assess the mechanical properties of the prepared gels TA.XTPlus measurements were conducted before and after exposure to buffer perfusion in the vFTC over a period of up to 28 days. Stress-strain curves of the reference (alginate) after sample compression of $25 \%$ without fracture are given in (Figure 4A). Experimental data were obtained before, after 1 and 2 days of buffer perfusion within the vFTC (individual gel for each experiment). Stress-strain curves of the reference after repeated compression are given in (Figure 4B).

In general, stress-strain curves of the reference are characterized by an initial approximately linear rise followed by a much steeper rise until reaching the maximal degree of sample compression (loading curve). The unloading curve is characterized by a similar shape but with a steeper initial decline thus forming a hysteresis loop. Maximum values of hardness of $9.5 \mathrm{kPa}$ at $\mathrm{t}=0,7.6 \mathrm{kPa}$ at $\mathrm{t}=1 \mathrm{~d}$ and $5.0 \mathrm{kPa}$ at $\mathrm{t}=2 \mathrm{~d}$ (Figure 4A) were recorded.
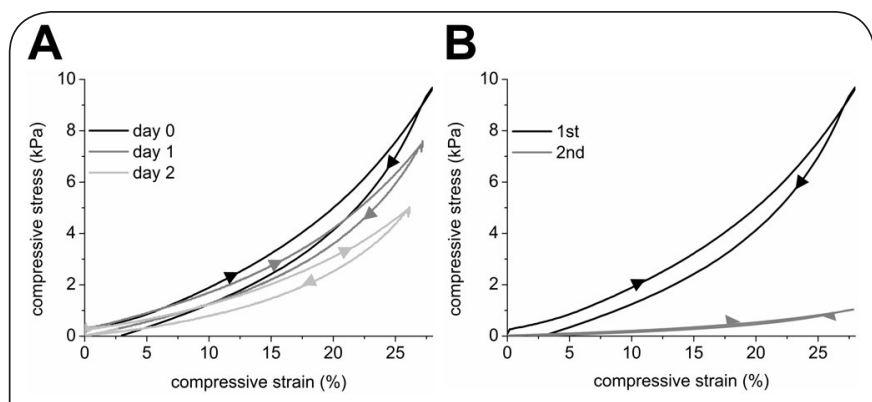

Figure 4. Typical plot of stress-strain data for alginate gels using texture analyzer TA.XTPlus obtained before and after 1 and 2 days buffer perfusion (A) and of two consecutive texture analyses on day 0 (B) (time between measurements: $1 \mathrm{~min}$ ), arrows indicating the loading $\downarrow$ and unloading $\triangleleft$ curves.

Hardness of the reference decreased from $9.5 \mathrm{kPa}$ ( $1^{\text {st }}$ loading) to $1.0 \mathrm{kPa}$ ( $2^{\text {nd }}$ loading) when sample compression was repeated after $1 \mathrm{~min}$ (Figure 4B). Additionally, the $2^{\text {nd }}$ loading curve possesses a completely different shape without any detectable hysteresis compared to the stress-strain profile of the $1^{\text {st }}$ compression. Alginate gels showed signs of erosion in the vFTC as early as after 3 days of buffer perfusion. Therefore, texture analysis was discontinued. After 7 days of buffer perfusion the gels were completely liquefied. Stress-strain curves of the test gels at a maximal deformation value of approximately 35\% without fracture are given in (Figure 5).

The shape of the stress-strain curves (individual gel for each experiment) of agar and agarose after 0 and 28 days of perfusion resembles the shape of the alginate curve spanning a hysteresis area. In comparison, the slope of the non-linear portions of the PVA curves is much less steep and the loading and unloading curves are very similar thus showing hardly 

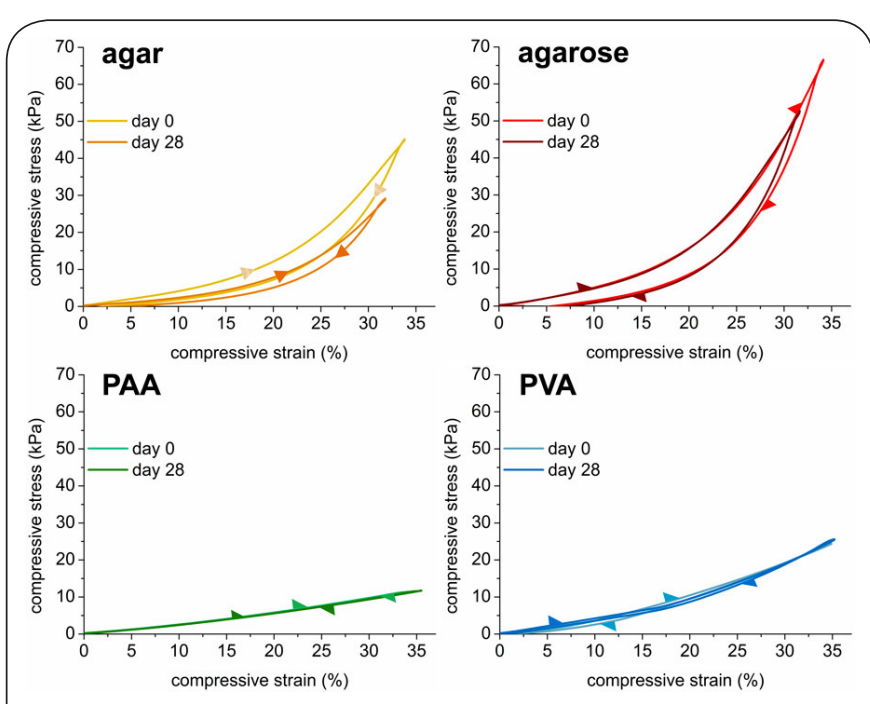

Figure 5.Typical plots of stress-strain data for agar, agarose, PAA and PVA gels using texture analyzer TA.XTPlus obtained before and after 28 days buffer perfusion, arrows indicating the loading $\downarrow$ and unloading $\triangleleft$ curves.

any hysteresis. Stress-strain curves of PAA are almost linear over the whole deformation range and loading and unloading curves are identical. Comparing the stress-strain profiles of native and perfused test gels of either PAA or PVA yielded no distinct differences. In the case of agarose gels, the loading and unloading curves before and after perfusion are also very similar but the gels differed regarding their hardness. This is most likely due to minimal differences in the sample height since $35 \%$ sample deformation was not reached in the 28 day sample. In the case of the agar gels, the loading curve at day 28 showed a slower rise compared to the loading curve at day 0 and the area between the curves was smaller. Hardness of agar at day 0 and at day 28 was $45.2 \mathrm{kPa}$ and $29.2 \mathrm{kPa}$, respectively, while for agarose $66.6 \mathrm{kPa}(\mathrm{t}=0)$ and $52.7 \mathrm{kPa}(\mathrm{t}=28 \mathrm{~d})$ were determined. Hardness of PVA ( $24.5 \mathrm{kPa}$ at $\mathrm{t}=0$ and $24.6 \mathrm{kPa}$ at $\mathrm{t}=28 \mathrm{~d}$ ) and PAA (11.4 $\mathrm{kPa}$ in both cases) did not change during the perfusion period of 28 days. To evaluate material fatigue, hydrogels were subjected to two consecutive compressions on day 0 . Experimental results are given in (Figure 6).

Two consecutive texture analyses of the identical gel cylinders demonstrated that hardness of agar and agarose gels was slightly lower in the second measurement. In detail, hardness of agar was lowered from $45.2 \mathrm{kPa}$ ( $1^{\text {st }}$ loading) to $40.0 \mathrm{kPa}$ ( $2^{\text {nd }}$ loading) at $\mathrm{t}=0$ and from $29.2 \mathrm{kPa}$ ( $1^{\text {st }}$ loading) to $22.7 \mathrm{kPa}$ ( $2^{\text {nd }}$ loading) at $\mathrm{t}=28 \mathrm{~d}$ (data not shown). For agarose gels loss of hardness from $66.6 \mathrm{kPa}$ (1 $1^{\text {st }}$ loading) to $60.7 \mathrm{kPa}\left(2^{\text {nd }}\right.$ loading) at $\mathrm{t}=0$ and from $52.5 \mathrm{kPa}$ ( $1^{\text {st }}$ loading) to $38.7 \mathrm{kPa}$ ( $2^{\text {nd }}$ loading) at $\mathrm{t}=28 \mathrm{~d}$ was observed. In comparison, no distinct change in hardness either at day $0\left(1^{\text {st }}\right.$ loading: $11.4 \mathrm{kPa}$ (PAA) and $24.5 \mathrm{kPa}(\mathrm{PVA}), 2^{\text {nd }}$ loading: $11.4 \mathrm{kPa}(\mathrm{PAA})$ and $24.5 \mathrm{kPa}$
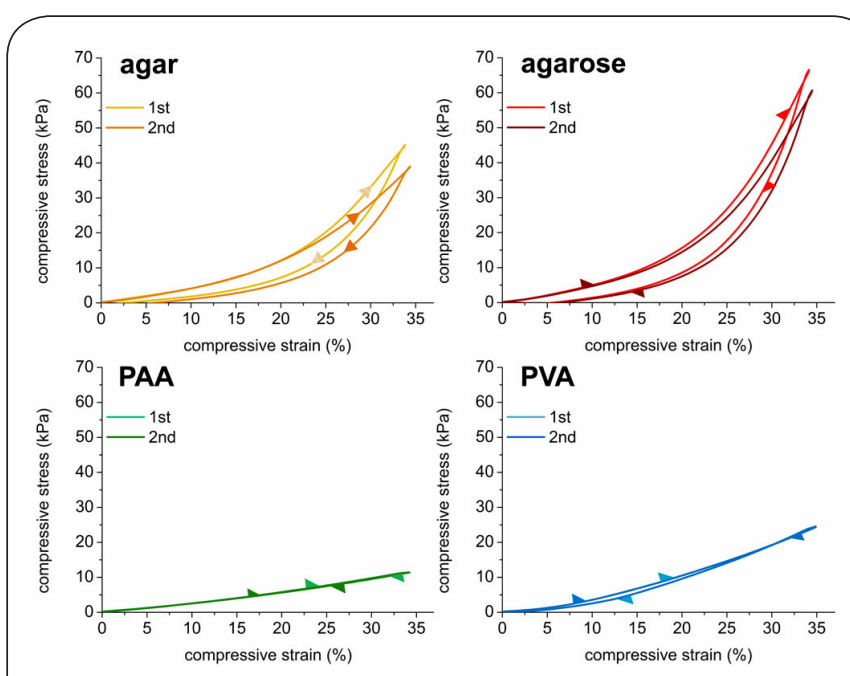

Figure 6. Typical plots of stress-strain data for agar, agarose, PAA and PVA gels using texture analyzer TA.XTPlus obtained during two consecutive texture analyses on day 0 (time between measurements: $1 \mathrm{~min}$ ), arrows indicating the loading $\downarrow$ and unloading 4 curves.
(PVA)) or at day $28\left(1^{\text {st }}\right.$ loading: $11.7 \mathrm{kPa}(\mathrm{PAA})$ and $25.6 \mathrm{kPa}$ (PVA), $2^{\text {nd }}$ loading: $11.6 \mathrm{kPa}(\mathrm{PAA})$ and $\left.25.8 \mathrm{kPa}(\mathrm{PVA})\right)$ for PAA and PVA was observed when repeating the texture analysis with the same gel cylinder.

The calculated Young's moduli of the reference and test gels are given in (Table 2).

Table 2. Young's moduli E (MPa) of the reference and the test gels calculated separately from individual stress-strain curves using equation 2.

\begin{tabular}{|c|c|c|c|c|c|}
\hline \multirow[t]{2}{*}{ day } & \multicolumn{4}{|c|}{$\mathrm{E}(\mathrm{MPa})$} & \multirow[b]{2}{*}{ PVA } \\
\hline & alginate & agar & agarose & PAA & \\
\hline \multirow[t]{2}{*}{0} & 0.017 & 0.040 & 0.049 & 0.023 & 0.033 \\
\hline & 0.019 & 0.040 & 0.049 & 0.023 & 0.032 \\
\hline \multirow[t]{2}{*}{1} & 0.014 & - & - & - & - \\
\hline & 0.014 & & & & \\
\hline \multirow[t]{2}{*}{2} & 0.010 & - & - & - & - \\
\hline & 0.009 & & & & \\
\hline \multirow[t]{2}{*}{28} & - & 0.021 & 0.045 & 0.022 & 0.040 \\
\hline & & 0.023 & 0.046 & 0.018 & 0.042 \\
\hline
\end{tabular}

Values of Young's moduli given in bold were calculated form the presented stress-strain curves. The second value was calculated form the non-depicted stress-strain curve $(n=2$ per gel formulation and time point).

Young's moduli of alginate, agar and PVA changed during the duration of buffer perfusion whereas Young's moduli of agarose and PAA remained approximately constant. Depending on type of gel formulation E varied from $0.017 / 0.019 \mathrm{MPa}$ 
(alginate) to $0.049 / 0.049 \mathrm{MPa}$ (agarose) at day 0 and from $0.021 / 0.023 \mathrm{MPa}$ (agar) to $0.045 / 0.046 \mathrm{MPa}$ (agarose) at day 28 , respectively. Alginate showed by far the lowest initial Young's moduli which were further decreased from $t=1 \mathrm{~d}$ to $\mathrm{t}=2 \mathrm{~d}$. E calculated for agarose samples provided the highest initial Young's moduli. Only very small deviations between the Young's moduli of the two samples measured at each time point were observed.

\section{Discussion}

The hydrogel used for biorelevant DES testing using the vFTC has to meet certain mechanical requirements. Form-stability of the cylindrical test specimen especially under perfusion of the vessel-simulating lumen at a fairly high flow rate for at least 30 days is of main importance. Furthermore, a short time span of gel-formation (or, in general, of sample preparation), transparency of the gels, elasticity, negligible swelling/shrinkage, diffusibility and biocompatibility are highly desirable.

\section{Preparation technique and optical characterization of test specimen}

With the exception of PVA the preparation of reference and test gels was easy and fast as only a few working steps were necessary. The solidification process of the thermally gelled polymer solutions of agar and agarose is caused by an increasing number of hydrogen bonds between molecules of neighboring polymer chains when the polymer coils become ordered as the solution is cooled [28]. In the case of the chemically cross-linked PAA gels, the cross-linking density is controlled by the amount of crosslinker as well as the ratio of acrylamide to $\mathrm{N}, \mathrm{N}^{\prime}$-methylene-bis-acrylamide [5]. Accordingly, the observed brittleness of PAA gels containing more than $10 \mathrm{wt} \%$ of the Rotiphorese gel 30 may be due to a denser polymeric network structure and resulting in breakage of the gel during removal from the vFTC. Using $10 \mathrm{wt} \%$ of the monomer/dimer stock solution of an acrylamide/ bis-acrylamide ratio of 37.5:1 seems appropriate to attain form-stable gels. The process of network densification upon freezing and thawing of PVA is caused by the formation of microcrystalline structures in the polymer solution, hydrogen bonds and phase separation (liquid - polymer fraction) during freezing and thawing $[\mathbf{2 4}, \mathbf{2 9}]$. Mechanical properties such as elasticity [30], tensile moduli [29], swelling [29,31] dissolution $[32]$ and pore size $[33,34]$ are a function of the percentage of the PVA solution and the number of cryogelation steps.

To facilitate balloon catheter/stent placement sample transparency is preferable. Clear and transparent test specimens were observed in the case of PAA. The cooling process during gel-formation of agar and agarose is accompanied by increasing turbidity. The formation of hydrogen bonds may be the cause of this as reported by Gelfi et al., for PAA gels formed under cooling [35]. As indicated by optical density measurements light absorbance is increased in $1 \mathrm{wt} \%$ agarose gels compared to gels containing $0.5 \mathrm{wt} \%$ polymer [36]. For PVA, the observed effect of sample whitening is affected by the formation of microcrystalline structures and consequently increases with each freeze-thaw step [24,29].

Pore size can be influenced by the polymer concentration. For agar gels (3-30 wt\%) it has been observed that gel density increased and pore size decreased with increasing content of the gelling agent [37]. A larger PVA fraction also leads to an increased number of smaller pores which are separated by thicker bridges of the cryogel structure [34]. Lira et al., reported that the pore size of PAA gels decreases until nonporous gels are attained with increasing polymer fraction either of acrylamide/bis-acrylamide or of bis-acrylamide [5]. This is, however, not the case with the gels prepared here, even though pores are not clearly visible in the SEM images. First experiments showed diffusion of dissolved model substances into all gels including the PAA gels (data not shown).

\section{Degree of swelling}

Taking target gel parameters into consideration sample swelling should be ideally negligible after gelation to exclude changes in the dimension of the gel cylinder especially of the hydrogel lumen. The experimental results indicate that this was not the case with regard to the reference, PAA and PVA. Nevertheless, swelling/shrinkage behavior did not affect perfusion of dissolution media in the vFTC as observed lumen renarrowing was negligibly small.

The theory of equilibrium swelling of cross-linked hydrogels was developed by Flory and Rehner in 1943 [38]. Here, swelling is understood as a balance between elasticity of the gel formulation and the evolved opposite osmotic pressure of the liquid phase. The maximum degree of swelling is limited by the numbers of cross-links, and the extent of swelling is inversely proportional to the density of the polymeric network [38]. However, prepared agar and agarose samples appear to be non-swell- or shrinkable. This may be explained by the assumption that the gel system is balanced between negative enthalpy (dissolution of polymer in the aqueous phase) and positive entropy (expansion/organization of dissolved polymer chains), i.e., native gel formulations of agar and agarose with a polymer fraction of $2 \mathrm{wt} \%$ have finally reached state of energetic equilibrium. Accordingly, the studied alginate and PAA gels had not reached a balanced water to polymer ratio resulting in changes during incubation. The degree of water uptake of cryogels is influenced by the number of freezing-thawing cycles $[\mathbf{2 3}, \mathbf{2 9}, \mathbf{3 1}]$ as well as the initial polymer content of the solution. The observed loss in sample weight may be related to dissolution of PVA chains which are not incorporated in the physical network. However, differences between expected and detected degree of swelling can only be partly explained by this as only a fractional dissolution of $0.3-0.5 \mathrm{wt} \%$ of various cryogels was observed by Hassan et al., [32].

Differences between the results of the swelling experiments 
and the observations made during perfusion in the vFTC for 28 days may be attributed to the differences in the test setups used. These differences are very obvious for alginate which was swollen in the incubation setup but showed signs of erosion in the vFTC. This might be caused by different volumes of media that were used ( $100 \mathrm{~mL}$ vs. $350 \mathrm{~mL}$ ) providing more volume for $\mathrm{Ca}^{2+}$ ion washout in the vFTC. The replacement of $\mathrm{Ca}^{2+}$ ions from alginate gels by monovalent buffer ions is the most likely reason for gel erosion. Furthermore, the type of agitation (moderate shaking vs. perfusion through a small opening at a high flow-rate) most likely influenced the gel erosion. Swelling of gels in the vFTC was not observed in spite of the mass gain of PAA samples in the swelling experiments. However, changes in the general volume are mainly excluded in the vFTC due to the direct contact with the wall of the vFTC. Narrowing of the vessel-simulating lumen was also not observed.

\section{Long-term stability}

Texture analyses were conducted in order to evaluate longterm stability of prepared gels and to compare mechanical properties before and after 28 days perfusion.

Gel hardness decreased for alginate gels with increasing perfusion time. In addition, sample erosion was observed leading to liquefaction of alginate gels after 7 days. This is most likely caused by the displacement of the chelating agent $\left(\mathrm{Ca}^{2+}\right)$ by monovalent ions of the perfusion media. As shown by LeRoux et al., the compressive modulus of $2 \mathrm{wt} \%$ calcium alginate gels was reduced by $63 \%$ after $15 \mathrm{~h}$ exposure to aqueous media containing physiological concentrations of sodium chloride compared to media without sodium chloride [39]. Furthermore, time dependence of gel softening is highly affected by the ionic strength of buffered media and the interval of media replacement as reported for alginate-based films by Livnat et al., [40]. Highly concentrated buffer-salt media and a narrow replacement interval will accelerate the process of sample erosion which finally leads to material failure of alginate gels in the vFTC. However, the experimental data on mechanical properties of gelled solutions of alginate and agarose after a prolonged buffer exposure using a static incubation setup by Shoichet et al., [8] do not completely match the data outcome under flow conditions of the work presented here. Shoichet et al., reported that gel stability of 1.5 wt $\%$ alginate gels decreased within the first 30 days [8]. In their study, gel stability of $1 \mathrm{wt} \%$ agarose gels was also lowered due to microbial growth and a related reduction in framework stability by interference with hydrogen bonding. In our experiments the measured texture properties of agarose did not change considerably, only in the case of agar distinct differences were observed. Although, microbial growth cannot be ruled out, we assume that softening of agar gels is related to spontaneous hydrolysis of the polymeric backbone of polysaccharide chains. Agarose profiles indicate long-term stability since loading and unloading curves of agarose profiles at day 0 match the TA.XTPlus profile at day 28 (identical shapes) with approximately identical hysteresis area. Slightly decreased hardness (day 0 vs. day 28) may be attributed to minimum differences in sample heights so identical sample deformation could not be reached. As no softening for PAA and PVA gels was observed these candidates are suitable for prolonged test periods in the VFTC. This phenomena for PAA (day 0 vs. day 28) is in agreement with Sun et al., who studied deformation behavior of alginate, alginate/PAA hybrid and PAA gels [3]. In this study, no differences were observed between loading and unloading curves of PAA indicating high elasticity of the network structure without breakage of junction points.

Time dependent rebuilding of network structure was observed for alginate, agar and agarose gels. The observed hysteresis of these test gels may be caused by a positional change of the junction points between polymer chains (alginate [3]) or by breaking hydrogen bonds of polymer chains (agar, agarose).

When comparing stress-strain curves of day $0 /$ day 28 with TA.XTPlus profiles obtained after $1^{\text {st }}$ loading $/ 2^{\text {nd }}$ loading at day 0 comparable tendencies for the hydrogel formulations are notable. Alginate and agar gels were softened after 28 days buffer perfusion, comparable to the degree of sample softening observed during the $2^{\text {nd }}$ compression step at day 0 . Moreover, loading and unloading curves of the $2^{\text {nd }}$ loading differ from the first loading and less compressive stress (or force) was required to cause the same percentage of gel deformation. Stress-strain behavior and value of hardness at $35 \%$ sample deformation of PAA and PVA gels remained almost unchanged after long term perfusion as well as repeated compression. In the case of agarose, slight differences between the $1^{\text {st }}$ and $2^{\text {nd }}$ loading were observed. However, no differences of the stress-strain curves between the $1^{\text {st }}$ loading at day 0 and day 0/day 28 were detected.

No apparent changes in Young's moduli of test specimens were observed for alginate, agarose and PAA. E of agar decreased and $E$ of PVA increased with exposure to flowing PBS pH 7.4. In the first case, elasticity will increase due to hydrolysis of the polysaccharide chains corresponding to its lowered hardness. The results of E for PVA are in accordance with the amount of desorbed water of the respective gel formulation. In theory, gel shrinkage will result in denser polymeric networks leading to hardening of the test specimen. In addition, as demonstrated by Alcantâra et al., of PVA gels were lower for samples of higher cross-linking density independent of the preparation technique and the gel composition used [41]. The complexity of cardiac tissue or human vessel wall components regarding mechanical properties has been reported by Bank et al., [42]. Experimental data varies over a wide range depending on different vessel wall structures (collagen, elastin and smooth muscle) which are either reported as elastic (elastin/collagen) or contractile (smooth muscle) components depending on the smooth muscle tone (fully relaxed, relaxed or fully contracted) and the 
applied pressure (50 or $100 \mathrm{mmHg}$ ). As gel compartments are intended to be used in the vFTC setup in order to allegorize some properties of the cardiac tissue it might be useful to mimic vessel wall elasticity. However, literature data vary in wide range indicating discrepancy of reported results obtained by different authors and setups and are hardly comparable $[42,43]$. In consequence, no target value for the Young's moduli can clearly be defined. All prepared gels are elastic and provide the choice of varying Young's moduli for biorelevant dissolution testing.

Even though texture analysis seems a useful approach to studying the material properties of hydrogels, further experiments will have to be undertaken to verify the suitability of the method and to be able to make statistically valid predictions of the long-term stability of the prepared test specimens based on this method.

Regarding target gel parameters, especially the stability of the prepared gel formulations under flow conditions, the performance of all test candidates (with the exception of alginate and agar) was sufficient. The results obtained from this study indicate that the investigation of drug release and distribution from DES which typically release their drug load over a prolonged period of time ( 90 days) becomes feasible using the newly developed gels. To further improve predictability of drug release by in vitro dissolution testing the entrapment of proteins and binding structures $[44,45]$ in the gelled compartment could be a useful approach. For this purpose, especially agar or agarose seem to be rather advantageous due to their mild gelling conditions compared to PAA and PVA gels. Gelled PAA samples are noteworthy for their mechanical properties. Hardness and elasticity were found to be completely unaffected by environmental conditions within vFTC over 28 days. Long-term stability of the cryogel formulation was suitable, however, the preparation technique is quite laborious.

\section{Conclusion}

Methods of gel preparation for the vFTC setup were established successfully for four test gels containing $2 \mathrm{wt} \%$ agar, $2 \mathrm{wt} \%$ agarose, $10 \mathrm{wt} \%$ PAA or $15 \mathrm{wt} \%$ PVA $(145.000 \mathrm{~g} / \mathrm{mol}, 5$ freezethaw cycles). Agarose samples seem to be the most suitable candidate for long-term dissolution testing since target gel parameters which are relevant for their use as a tissuesimulating compartment in the vFTC were met: the gel formulation leads to form-stable gel compartments for the vFTC which retain their cylindrical shape for periods of at least 28 days, gels are sufficiently transparent in order to allow monitoring of DES placement in the gel lumen, gels are diffusible, swelling is negligible small, gels are elastic, and the composition allows for the inclusion of cells. Texture measurements confirmed sufficient mechanical stability of the prepared gels under long-term test conditions for 28 days. Gels prepared of agar do not exactly comply with the same requirements as agarose. Sample softening was observed after
28 days of perfusion. PAA and PVA samples also showed longterm mechanical stability. None of the other test specimen fulfilled all target gel parameters, however, each formulation may be useful to clarify drug diffusion characteristics and characterize drug release from DES in vitro using the vFTC. Further research will be undertaken to investigate possible variation in transport properties of the developed gels.

\section{Competing interests}

The authors declare that they have no competing interests. Authors' contributions

\begin{tabular}{|l|l|l|l|l|l|}
\hline Authors' contributions & BS & SN & KS & WW & AS \\
\hline Research concept and design & $\checkmark$ & -- & $\checkmark$ & $\checkmark$ & $\checkmark$ \\
\hline Collection and/or assembly of data & $\checkmark$ & -- & - & -- & - \\
\hline Data analysis and interpretation & $\checkmark$ & $\checkmark$ & -- & $\checkmark$ & $\checkmark$ \\
\hline Writing the article & $\checkmark$ & $\checkmark$ & -- & $\checkmark$ & $\checkmark$ \\
\hline Critical revision of the article & $\checkmark$ & $\checkmark$ & $\checkmark$ & $\checkmark$ & $\checkmark$ \\
\hline Final approval of article & $\checkmark$ & -- & -- & -- & $\checkmark$ \\
\hline
\end{tabular}

\section{Acknowledgement}

The authors would like to acknowledge the technical assistance of Romy Schütt. Additionally, the authors are deeply grateful to Dr. Barbara Dittrich for helpful suggestions regarding PVA gelation and to Jenny Bandomir for useful advices on the PAA gel preparation technique. This research was funded by the BMBF (Federal Ministry of Education and Research) within REMEDIS: "Höhere Lebensqualität durch neuartige Mikroimplantate".

\section{Publication history}

Editor: Narasimha Murthy S, The University of Mississippi, USA. EIC: George Perry, University of Texas at San Antonio, USA.

Received: 17-Aug-2013 Revised: 11-Oct-2013

Rerevised: 18-Oct-2013 Accepted: 09-Dec-2013

Published: 28-Dec-2013

\section{References}

1. Miyata $\mathrm{T}$, Asami $\mathrm{N}$ and Uragami T. A reversibly antigen-responsive hydrogel. Nature. 1999; 399:766-9. | Article | PubMed

2. Draget KI, Smidsrød O and Skjåk-Bræk G: Alginates from Algae. Biopolymers. 2005. | Article

3. Sun JY, Zhao X, Illeperuma WR, Chaudhuri O, Oh KH, Mooney DJ, Vlassak JJ and Suo Z. Highly stretchable and tough hydrogels. Nature. 2012; 489:133-6. | Article | PubMed Abstract | PubMed Full Text

4. Park TG and Hoffman AS. Synthesis and characterization of pH- and/or temperature-sensitive hydrogels. Journal of Applied Polymer Science. 1992; 46:659-71. | Article

5. Lira LM, Martins KA and Torresi SICd. Structural parameters of polyacrylamide hydrogels obtained by the Equilibrium Swelling Theory. European Polymer Journal. 2009; 45:1232-8. | Article

6. Kirschner CM and Anseth KS. Hydrogels in Healthcare: From Static to Dynamic Material Microenvironments. Acta Mater. 2013; 61:931-944. I Article | PubMed Abstract | PubMed Full Text

7. Saul JM, Williams DF: Hydrogels in Regenerative Medicine. In Anthony A, Robert L, James AT, Nerem RM, editors. Principles of Regenerative Medicine 2011, 637-61.

8. Shoichet MS, Li RH, White ML and Winn SR. Stability of hydrogels used in cell encapsulation: An in vitro comparison of alginate and agarose. Biotechnol Bioeng. 1996; 50:374-81. | Article I PubMed

9. Lee HR, Park KM, Joung YK, Park KD and Do SH. Platelet-rich plasma loaded hydrogel scaffold enhances chondrogenic differentiation and maturation with up-regulation of CB1 and CB2. J Control Release. 2012; 
159:332-7. | Article | PubMed

10. Terry CM, Li L, Li H, Zhuplatov I, Blumenthal DK, Kim SE, Owen SC, Kholmovski EG, Fowers KD, Rathi $R$ and Cheung AK. In vivo evaluation of the delivery and efficacy of a sirolimus-laden polymer gel for inhibition of hyperplasia in a porcine model of arteriovenous hemodialysis graft stenosis. J Control Release. 2012; 160:459-67. | Article | PubMed Abstract | PubMed Full Text

11. Peppas NA, Bures $P$, Leobandung $W$ and Ichikawa $H$. Hydrogels in pharmaceutical formulations. Eur J Pharm Biopharm. 2000; 50:27-46. I Article | PubMed

12. Klose D, Azaroual N, Siepmann F, Vermeersch G and Siepmann J. Towards more realistic in vitro release measurement techniques for biodegradable microparticles. Pharm Res. 2009; 26:691-9. | Article | PubMed

13. Hoang Thi TH, Chai F, Lepretre S, Blanchemain N, Martel B, Siepmann F, Hildebrand HF, Siepmann J and Flament MP. Bone implants modified with cyclodextrin: study of drug release in bulk fluid and into agarose gel. Int J Pharm. 2010; 400:74-85. | Article | PubMed

14. Neubert A, Sternberg K, Nagel S, Harder C, Schmitz KP, Kroemer HK and Weitschies W. Development of a vessel-simulating flow-through cell method for the in vitro evaluation of release and distribution from drug-eluting stents. J Control Release. 2008; 130:2-8. | Article | PubMed

15. O'Brien CC, Finch CH, Barber TJ, Martens P and Simmons A. Analysis of drug distribution from a simulated drug-eluting stent strut using an in vitro framework. Ann Biomed Eng. 2012; 40:2687-96. | Article | PubMed

16. Seidlitz A, Nagel S, Semmling B, Grabow N, Martin H, Senz V, Harder C, Sternberg K, Schmitz KP, Kroemer HK and Weitschies W. Examination of drug release and distribution from drug-eluting stents with a vesselsimulating flow-through cell. Eur J Pharm Biopharm. 2011; 78:36-48. | Article | PubMed

17. Food and Drug Administration. Guidance for industry: Dissolution Testing of Immediate Release Solid Oral Dosage Forms. Rockville, MD: US Department of Health and Human Services, FDA, Center for Drug Evaluation and Research, 1997.

18. Tzafriri AR, Groothuis A, Price GS and Edelman ER. Stent elution rate determines drug deposition and receptor-mediated effects. J Control Release. 2012; 161:918-26. | Article | PubMed Abstract | PubMed Full Text

19. Balakrishnan B, Dooley JF, Kopia G and Edelman ER. Intravascular drug release kinetics dictate arterial drug deposition, retention, and distribution. J Control Release. 2007; 123:100-8. | Article | PubMed Abstract | PubMed Full Text

20. Cypher ${ }^{\circ}$ Sirolimus-eluting Coronary Stent. Miami: Cordis Corporation; 2008.

21. Draget KI, Stokke BT, Yuguchi Y, Urakawa H and Kajiwara K. Small-angle $\mathrm{X}$-ray scattering and rheological characterization of alginate gels. 3. Alginic acid gels. Biomacromolecules. 2003; 4:1661-8. | Article | PubMed

22. Hurler J, Engesland A, Poorahmary Kermany B and Škalko-Basnet $\mathrm{N}$. Improved texture analysis for hydrogel characterization: Gel cohesiveness, adhesiveness, and hardness. Journal of Applied Polymer Science. 2012; 125:180-8. | Article

23. Peppas NA and Scott JE. Controlled release from poly(vinyl alcohol) gels prepared by freezing-thawing processes. Journal of Controlled Release. 1992; 18:95-100. | Article

24. Hassan CM and Peppas NA. Structure and Applications of Poly(vinyl alcohol) Hydrogels Produced by Conventional Crosslinking or by Freezing/Thawing Methods. Biopolymers PVA Hydrogels, Anionic Polymerisation Nanocomposites. 2000; 37-65. | Pdf

25. Di Mario C, Meneveau N, Gil R, de Jaegere P, de Feyter PJ, Slager CJ, Roelandt JR and Serruys PW. Maximal blood flow velocity in severe coronary stenoses measured with a Doppler guidewire. Limitations for the application of the continuity equation in the assessment of stenosis severity. Am J Cardiol. 1993; 71:54D-61D. | Article | PubMed

26. Pons $M$ and Fiszman SM. Instrumental texture profile analysis with particular reference to gelled systems. Journal of Texture Studies. 1996; 27:597-624. | Article

27. Szczesniak AS. Classification of Textural Characteristicsa. Journal of Food Science. 1963; 28:385-9. | Article

28. Braudo EE, Muratalieva IR, Plashchina IG, Tolstoguzov VB and Markovich IS. Studies on the mechanisms of gelation of kappa-carrageenan and agarose. Colloid and Polymer Science. 1991; 269:1148-56. | Article |

29. Holloway JL, Lowman AM and Palmese GR. Aging behavior of PVA hydrogels for soft tissue applications after in vitro swelling using osmotic pressure solutions. Acta Biomater. 2013; 9:5013-21. | Article | PubMed

30. Duboeuf F, Basarab A, Liebgott H, Brusseau E, Delachartre P and Vray D. Investigation of PVA cryogel Young's modulus stability with time, controlled by a simple reliable technique. Med Phys. 2009; 36:656-61. I Article | PubMed

31. Fumio U, Hiroshi Y, Kumiko N, Sachihiko N, Kenji S and Yasunori M. Swelling and mechanical properties of poly(vinyl alcohol) hydrogels. International Journal of Pharmaceutics. 1990; 58:135-42. | Article

32. Hassan CM, Trakampan P, Peppas NA: Water Solubility Characteristics of Poly(vinyl alcohol) and Gels Prepared by Freezing/Thawing Processes. Water Soluble Polymers. 2002; 31-40. | Article

33. Willcox PJ, Howie DW, Schmidt-Rohr K, Hoagland DA, Gido SP and Pudjijanto $S$ et al.: Microstructure of poly(vinyl alcohol) hydrogels produced by freeze/thaw cycling. Journal of Polymer Science Part B: Polymer Physics. 1999; 37:3438-54. | Article

34. Fergg F, Keil FJ and Quader H. Investigations of the microscopic structure of poly(venyl alcohol) hydrogels by confocal laser scanning microscopy. Colloid Polymer Science. 2001; 279:61-7. | Article

35. Gelfi C and Righetti PG. Polymerization kinetics of polyacrylamide gels II. Effect of temperature. Eelectrophoresis. 1981; 2:220-8. I Article

36. Jaaky $\mathrm{N}$, Junying $\mathrm{X}$ and Xiangyang L. Determination of agarose gel pore size: Absorbance measurements vis a vis other techniques. Journal of Physics. 2006; 28:83-6. | Article

37. Ayyad O, Munoz-Rojas D, Agullo N, Borros S and Gomez-Romero P. Highconcentration compact agar gels from hydrothermal synthesis. Soft Matter. 2010; 6:2389-91. | Article

38. Flory PJ and Rehner JJ. Statistical Mechanics of Cross-Linked Polymer Networks II. Swelling. The Journal of Chemical Physics. 1943; 11:521-6. | Article

39. LeRoux MA, Guilak F and Setton LA. Compressive and shear properties of alginate gel: effects of sodium ions and alginate concentration. J Biomed Mater Res. 1999; 47:46-53. | Article | PubMed

40. Livnat M, Beyar R and Seliktar D. Endoluminal hydrogel films made of alginate and polyethylene glycol: physical characteristics and drugeluting properties. J Biomed Mater Res A. 2005; 75:710-22. | Article | PubMed

41. Alcântara MTS, Brant AJC, Giannini DR, Pessoa JOCP, Andrade AB and Riella HG et al. Influence of dissolution processing of PVA blends on the characteristics of their hydrogels synthesized by radiation-Part I: Gel fraction, swelling, and mechanical properties. Radiation Physics and Chemistry. 2012; 81:1465-70. | Article

42. Bank AJ, Wang H, Holte JE, Mullen K, Shammas R and Kubo SH. Contribution of collagen, elastin, and smooth muscle to in vivo human brachial artery wall stress and elastic modulus. Circulation. 1996; 94:3263-70. | Article | PubMed

43. Bergel DH. The static elastic properties of the arterial wall. J Physiol. 1961; 156:445-57. | Article | PubMed Abstract | PubMed Full Text

44. Tzafriri $A R$, Levin $A D$ and Edelman ER. Diffusion-limited binding explains binary dose response for local arterial and tumour drug delivery. Cell Prolif. 2009; 42:348-63. | Article | PubMed Abstract | PubMed Full Text

45. Levin AD, Vukmirovic N, Hwang CW and Edelman ER. Specific binding to intracellular proteins determines arterial transport properties for rapamycin and paclitaxel. Proc Natl Acad Sci U S A. 2004; 101:9463-7. I Article | PubMed Abstract | PubMed Full Text

\section{Citation:}

Semmling B, Nagel S, Sternberg K, Weitschies W and Seidlitz A. Long-term stable hydrogels for biorelevant dissolution testing of drug-eluting stents. J Pharm Technol Drug Res. 2013; 2:19. http://dx.doi.org/10.7243/2050-120X-2-19 